\title{
ARTICLE
}

Received 10 Oct 2012 | Accepted 25 Feb 2013 | Published 9 Apr $2013 \quad$ DOl: 10.1038/ncomms2640

\section{Soft X-ray spectromicroscopy using ptychography with randomly phased illumination}

\author{
A.M. Maiden ${ }^{1, \dagger}$, G.R. Morrison ${ }^{2, \dagger}$, B. Kaulich ${ }^{3, \dagger}$, A. Gianoncelli ${ }^{3}$ \& J.M. Rodenburg ${ }^{1}$
}

Ptychography is a form of scanning diffractive imaging that can successfully retrieve the modulus and phase of both the sample transmission function and the illuminating probe. An experimental difficulty commonly encountered in diffractive imaging is the large dynamic range of the diffraction data. Here we report a novel ptychographic experiment using a randomly phased $\mathrm{X}$-ray probe to considerably reduce the dynamic range of the recorded diffraction patterns. Images can be reconstructed reliably and robustly from this setup, even when scatter from the specimen is weak. A series of ptychographic reconstructions at X-ray energies around the $L$ absorption edge of iron demonstrates the advantages of this method for soft X-ray spectromicroscopy, which can readily provide chemical sensitivity without the need for optical refocusing. In particular, the phase signal is in perfect registration with the modulus signal and provides complementary information that can be more sensitive to changes in the local chemical environment.

\footnotetext{
${ }^{1}$ Department of Electronic and Electrical Engineering, University of Sheffield, Sheffield S1 3JD, UK. ${ }^{2}$ Department of Physics, King's College London, Strand, London WC2R 2LS, UK. ${ }^{3}$ Elettra-Sincrotrone Trieste S.C.p.A., Basovizza, Trieste I-34149, Italy. `Present addresses: Phase Focus Ltd., Electric Works, Sheffield S1 2BJ, UK (A.M.M.); University College London, London Centre for Nanotechnology, 17-19 Gordon St., London WC1H OAH, UK; Research Complex at Harwell, Rutherford Appleton Laboratory, Didcot OX11 OFA, UK (G.R.M.); Diamond Light Source, Harwell Science and Innovation Campus, Didcot OX11 ODE, UK (B.K.). Correspondence and requests for materials should be addressed to G.R.M. (email: graeme.morrison@ucl.ac.uk).
} 
P tychography is rapidly developing into an important tool in $\mathrm{X}$-ray microscopy. The technique involves successively illuminating overlapping regions of a specimen with a localized probe and recording the resulting diffraction patterns. A key requirement is that the illuminated areas overlap substantially, so that iterative algorithms $\mathrm{s}^{1-3}$ can successfully reconstruct an image of the specimen, retrieving the amplitude and phase of both the complex sample transmission function and the illuminating probe. At X-ray wavelengths, ptychography has been used to characterize the focusing properties of X-ray optics $^{4-6}$, to image interconnects within microchips ${ }^{7}$ and to image yeast cells ${ }^{8}$. X-ray ptychography has recently been demonstrated using soft X-rays ${ }^{9,10}$, and has been used with polarized illumination to generate dichroic image contrast from magnetic thin films ${ }^{11}$. It can also be combined with tomography to produce three-dimensional reconstructions ${ }^{12-14}$.

Despite these successes, ptychographic imaging suffers from the same recurring problem as coherent diffraction imaging, in that the diffraction patterns generally have an extremely large dynamic range, with a zero-order component orders of magnitude more intense than the scattered signal. As a result, multiple exposures may be needed to allow accurate recording of the full dynamic range of the diffraction pattern or a beamstop may be introduced to block the zero-order component. However, as some ptychographic algorithms do not require a priori knowledge of the form of the illuminating probe, but can extract this information from the recorded data sets, it is possible to consider enhancing the phase diversity of the probe to reduce the dynamic range of the recorded diffraction data and improve the quality of the reconstructed image ${ }^{15,16}$. This approach is conceptually similar to the use of coded-aperture imaging at visible $^{17}$ and X-ray wavelengths ${ }^{18}$, except that the ptychographic approach does not require a priori knowledge of the coded apertures to achieve successful image reconstructions.

At visible-light wavelengths, a diffuser was used to randomize the phase structure of the ptychographic probe ${ }^{15}$. Here we demonstrate the method in the soft X-ray regime, reducing the dynamic range of the diffraction patterns by an order of magnitude. In this case, the phase diversity of the probe was enhanced by introducing into the beam path a pseudo-random array of $\sim 40 \mathrm{~nm}$ diameter holes in a 140-nm thick tungsten film. We show that despite the complexity of the probe wavefront, samples with relatively small X-ray-scattering cross-sections can be imaged successfully. A recent mathematical analysis has shown that randomly phased illumination can also help standard phasing algorithms converge more rapidly to a reliable solution ${ }^{19}$. We employ our novel experimental geometry to generate a series of ptychographic reconstructions at X-ray energies around the $\mathrm{L}$ absorption edge of iron, demonstrating chemical sensitivity without the need for optical refocusing. The amplitude and phase of the complex sample transmittance are recovered simultaneously by the ptychographic reconstructions, in perfect registration and at the same spatial resolution, allowing a major enhancement of the information normally available from near-edge X-ray spectromicroscopy. In particular, our results show that the phase signal can provide a more sensitive indication of changes in the local chemical environment.

\section{Results}

Description of the experiment. Experiments were carried out using the TwinMic X-ray microscope at the Elettra facility ${ }^{20}$. TwinMic has the unique capability to operate as both a transmission X-ray microscope (TXM) and as a scanning TXM (STXM). A hybrid mode involving both aspects was used for our ptychography experiments, as shown schematically in Fig. 1.

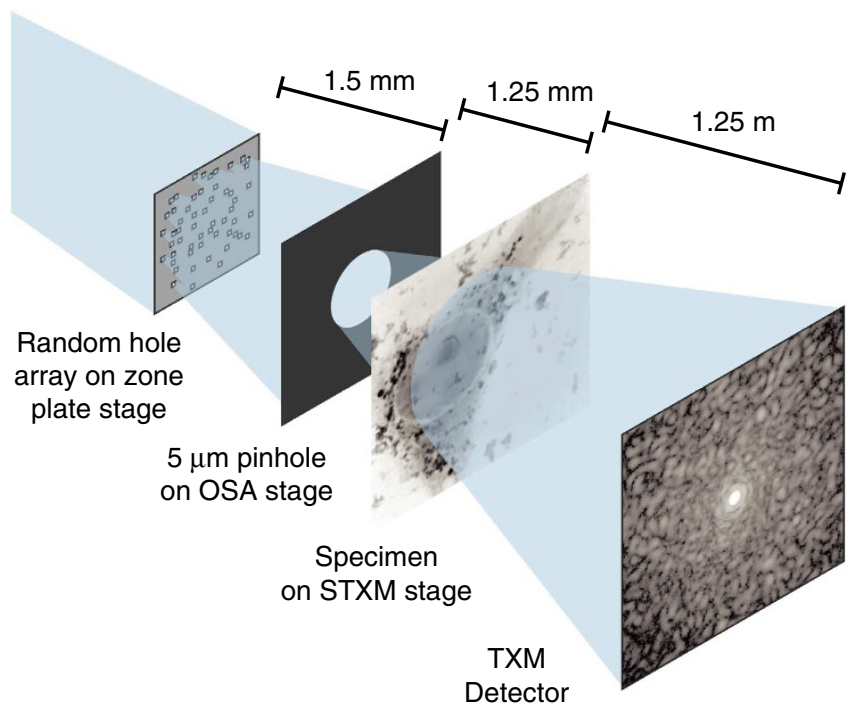

Figure 1 | Schematic representation of the modified experimental setup on the TwinMic beamline. A monochromatic $X$-ray beam illuminates an array of randomly positioned pinholes ( $\sim 40 \mathrm{~nm}$ diameter) that replaces the zone plate normally used during STXM operation of the TwinMic instrument. The pinholes act to diffuse the illumination and part of this diffuse beam is selected by a beam-defining pinhole that occupies the order-selecting aperture (OSA) stage used in STXM mode. The specimen sits on the standard STXM stage and is scanned across the beam in small rasters. The CCD normally used for imaging in TXM mode is used to record the diffraction pattern produced by the sample for every position in the raster scan.

To assess the effectiveness of the random hole array in reducing the dynamic range of the diffraction data, diffraction patterns were recorded from the $5-\mu \mathrm{m}$ probe-defining pinhole alone, and when the hole array was in position upstream of the $5-\mu \mathrm{m}$ pinhole. Three exposures were recorded in each case: at 1 , 0.5 and $0.1 \mathrm{~s}$ for the pinhole alone, and 10,5 and $1 \mathrm{~s}$ for the combination of the pinhole and the random hole array, which absorbed approximately $70 \%$ of the incident beam at energies around $700 \mathrm{eV}$. These exposures were combined to produce the results shown in Fig. 2, from which it is clear that the random hole array has significantly increased the fraction of the signal distributed across the detector plane, by about an order of magnitude. Figure 2a retains a relatively intense central spot, so that a significant further reduction in dynamic range could be achieved by improving our method of randomizing the phase in the pinhole plane.

Figure 3 shows the reconstructed modulus and phase of the specimen transmission function from a Balb/3T3 mouse fibroblast cell that had been doped with cobalt ferrite $\left(\mathrm{CoFe}_{2} \mathrm{O}_{4}\right)$ nanoparticles ${ }^{21}$. The set of diffraction patterns used for this reconstruction was collected at a beam energy of $708.8 \mathrm{eV}$ from a regular grid of $27 \times 27$ specimen positions, with a grid spacing of $1 \mu \mathrm{m}$. Clusters of $\mathrm{CoFe}_{2} \mathrm{O}_{4}$ particles appear as small dark spots in the modulus image, concentrated mainly in the perinuclear region, but are not visible in the phase reconstruction. This is consistent with the theoretical values tabulated by Chantler ${ }^{22,23}$ for the complex atomic X-ray-scattering factor at the iron L edge, which shows that the real part of the complex refractive index will have its minimum absolute value at around $708 \mathrm{eV}$, while this energy marks the onset of the absorption edge.

The incident X-ray probe is recovered simultaneously by the extended ptychographical iterative engine (ePIE) reconstruction ${ }^{3}$ and is shown in Fig. 4a, where brightness represents the 
amplitude and hue represents the phase. Propagating the probe upstream to the plane of the pinhole produces Fig. $4 \mathrm{~b}$ : the shape of the pinhole is well defined, with a diameter $\sim 5 \mu \mathrm{m}$, and the structure of the wavefront within it demonstrates the effectiveness of the random hole array as a diffusing mask.

One issue with our method is the difficulty of sufficiently localizing the probe at the plane of the sample, a problem also noted by Giewekemeyer et al. ${ }^{9}$. This was exacerbated in our case by the requirement to bin the diffraction data (see Methods section). Consequently a small degree of aliasing at the extremities of the probe reconstruction did produce some low intensity noise beyond the aperture of the pinhole that is too dim to be seen in Fig. 4b. The ptychographic reconstructions in Fig. 3a,b have an estimated resolution $\leqslant 250 \mathrm{~nm}$, which is significantly smaller than the probe size on the sample, and have levels of image detail and contrast that are directly comparable to the conventional STXM absorption
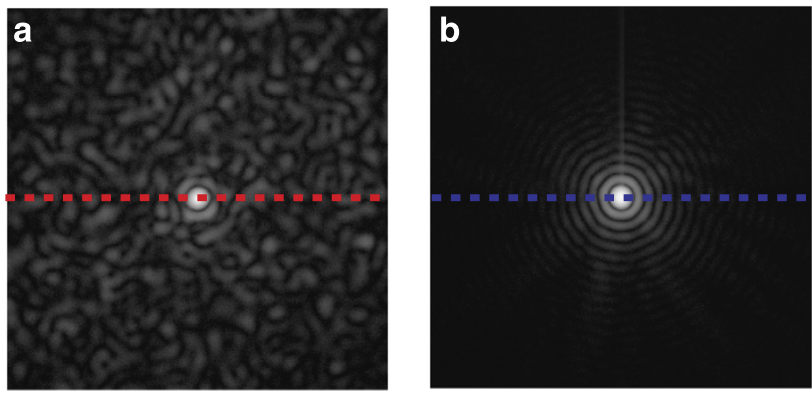

C

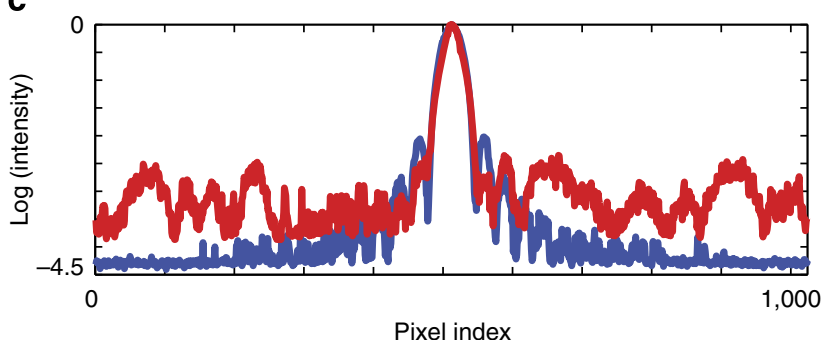

Figure 2 | Diffraction patterns recorded in the absence of a sample. The diffraction patterns in $\mathbf{a}$ and $\mathbf{b}$ were obtained by the combination of three different exposures and are shown with logarithmic scaling of the intensity. (a) The pattern obtained with the random-hole array in place upstream of the 5 - $\mu \mathrm{m}$ pinhole using exposures of 10,5 and $1 \mathrm{~s}$. (b) The pattern obtained with only the $5-\mu \mathrm{m}$ pinhole using exposures of $1,0.5$ and $0.1 \mathrm{~s}$. (c) Line plots of the intensity along the dashed lines in $\mathbf{a}$ and $\mathbf{b}$ image shown in Fig. 3c. This image was acquired using zone-plate optics to produce an X-ray probe size $\sim 120 \mathrm{~nm}$.

Imaging at energies around the iron $\mathrm{L}$ edge. Figure 5 shows ptychographic reconstructions from data collected at four other energies around the $\mathrm{Fe}_{3}$ edge. In these scans, the raster size was $18 \times 18$ and the grid spacing was $1.5 \mu \mathrm{m}$. The figure shows that the changes in image contrast from the $\mathrm{CoFe}_{2} \mathrm{O}_{4}$ particles are significantly different for the modulus and phase signals. Assuming the sample is sufficiently thin to be modelled by a multiplicative complex transmission function $h(\mathbf{r})=\exp [2 \pi i(n(\mathbf{r})-1) t(\mathbf{r}) / \lambda]$, where the complex refractive index $n(\mathbf{r})=1-\delta(\mathbf{r})+i \beta(\mathbf{r}), t(\mathbf{r})$ is the local thickness of the sample and $\lambda$ is the wavelength of the illumination, then the optical thickness $(n(\mathbf{r})-1) t(\mathbf{r})$ can be found directly from the ptychographic reconstructions. In particular, the phase image contrast is proportional to $-2 \pi \delta t / \lambda$, while the natural log of the modulus image gives $-2 \pi \beta t / \lambda$. In addition, the optical thickness can be separated into two components: one associated with the organic matter of the cell, and the other associated with the $\mathrm{CoFe}_{2} \mathrm{O}_{4}$ nanoparticles. The first of these components will be almost constant for small energy changes around the Fe L edge, so the difference between two phase images at nearby energies yields a signal that relates directly to the optical thickness of the $\mathrm{CoFe}_{2} \mathrm{O}_{4}$ nanoparticles. The same is true for the difference between two $\log _{e}$ (modulus) images taken at nearby energies.

Regions of interest (RoI) were defined by subtracting two phase images at nearby energies to produce a binary mask that could be applied to all the phase and $\log _{e}$ (modulus) images, allowing statistical data to be obtained for 66 RoI where the contrast was
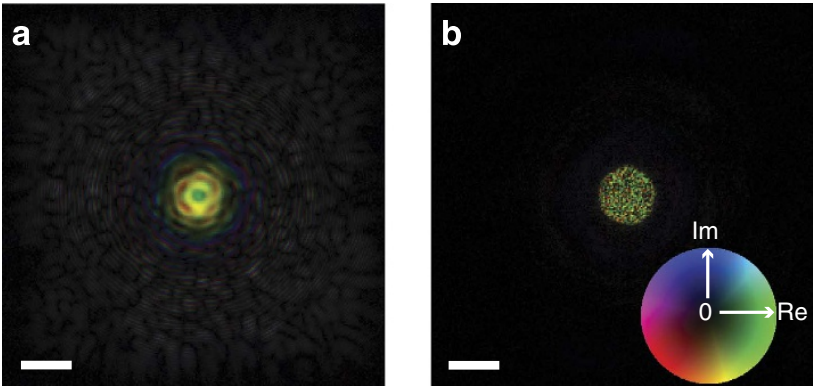

Figure 4 | Reconstructed probe wavefront. The real and imaginary parts of the reconstructed wavefront are shown using a colorwheel scale. (a) Reconstruction at the sample plane. (b) The wavefront after propagation upstream to the pinhole plane. Scale bar, $5 \mu \mathrm{m}$.
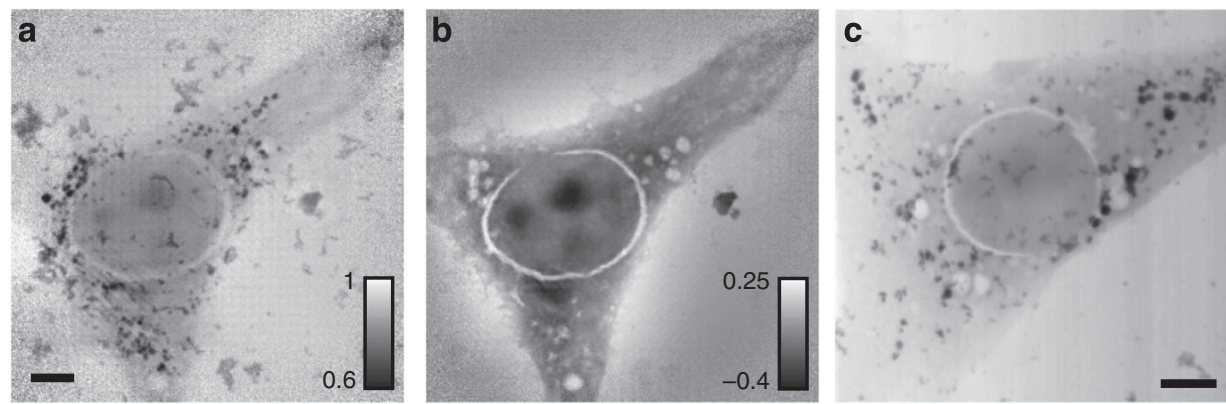

Figure 3 | Reconstructed modulus and phase images of a mouse Balb/3T3 fibroblast cell. The data for $\mathbf{a}$ and $\mathbf{b}$ were recorded at an X-ray energy of $708.8 \mathrm{eV}$. The modulus scale is in arbitrary units and the phase scale in radians. (a) Modulus image, where the clusters of $\mathrm{CoFe}_{2} \mathrm{O}_{4}$ nanoparticles appear as small dark spots. (b) Phase image, where the nanoparticle clusters have much lower contrast. (c) A conventional STXM absorption image of a similar mouse fibroblast cell taken at $707 \mathrm{eV}$ with an $\mathrm{X}$-ray probe size $\sim 120 \mathrm{~nm}$. Scale bar, $5 \mu \mathrm{m}$ (in a and $\mathbf{c}$ ). 
changing as a function of energy. The results of this analysis are plotted in Fig. 6, which shows the variation of $\log _{e}$ (modulus) and phase with energy for RoI that have been separated into five different classes. The values of $-2 \pi \beta t / \lambda$ and $-2 \pi \delta t / \lambda$ for $\mathrm{CoFe}_{2} \mathrm{O}_{4}$ calculated using Chantler's compilation of atomic scattering factors ${ }^{22,23}$ are plotted on the same graph, using a nominal $\mathrm{CoFe}_{2} \mathrm{O}_{4}$ thickness of $t=90 \mathrm{~nm}$ to ensure that the vertical scaling of the calculated data approximately matches the experimentally observed variations in the signals. To make comparison easier, a vertical offset has been added to our measurements of the class-average values for the RoI, so that they lie on the curves of Chantler's data at the lowest energy $(697.8 \mathrm{eV})$. To our knowledge, there are no published measurements of the phase variation across the $\mathrm{Fe} L$ edge for $\mathrm{CoFe}_{2} \mathrm{O}_{4}$, but measurements of the X-ray absorption spectrum and the X-ray magnetic circular dichroism signal have been made by Pattrick et al. ${ }^{24}$ and on $\mathrm{CoFe}_{2} \mathrm{O}_{4}$ nanoparticles by Coker et $a .^{25}$, who kindly provided their total electron yield (TEY) measurements of absorption across the Fe L edge (V. S. Coker, personal communication). Although it is difficult to make a direct comparison between TEY measurements and thin-film X-ray transmission data, a logarithmically scaled version of these TEY data is also plotted on Fig. 6 to provide a comparison with the $\log _{e}$ (modulus) data. The TEY data were also used to provide a more accurate energy calibration of the data available from the monochromator on the TwinMic beamline at Elettra. Average values for each class of the RoI are plotted in a separate colour in Fig. 6, and dots with the same colours identify the locations of the RoI on the inset phase image.

\section{Discussion}

We have shown how one of the major practical difficulties of $\mathrm{X}$-ray ptychography, the extremely large dynamic range of the diffraction patterns, can be alleviated at soft X-ray energies by introducing a suitable diffusing structure into the probe-forming
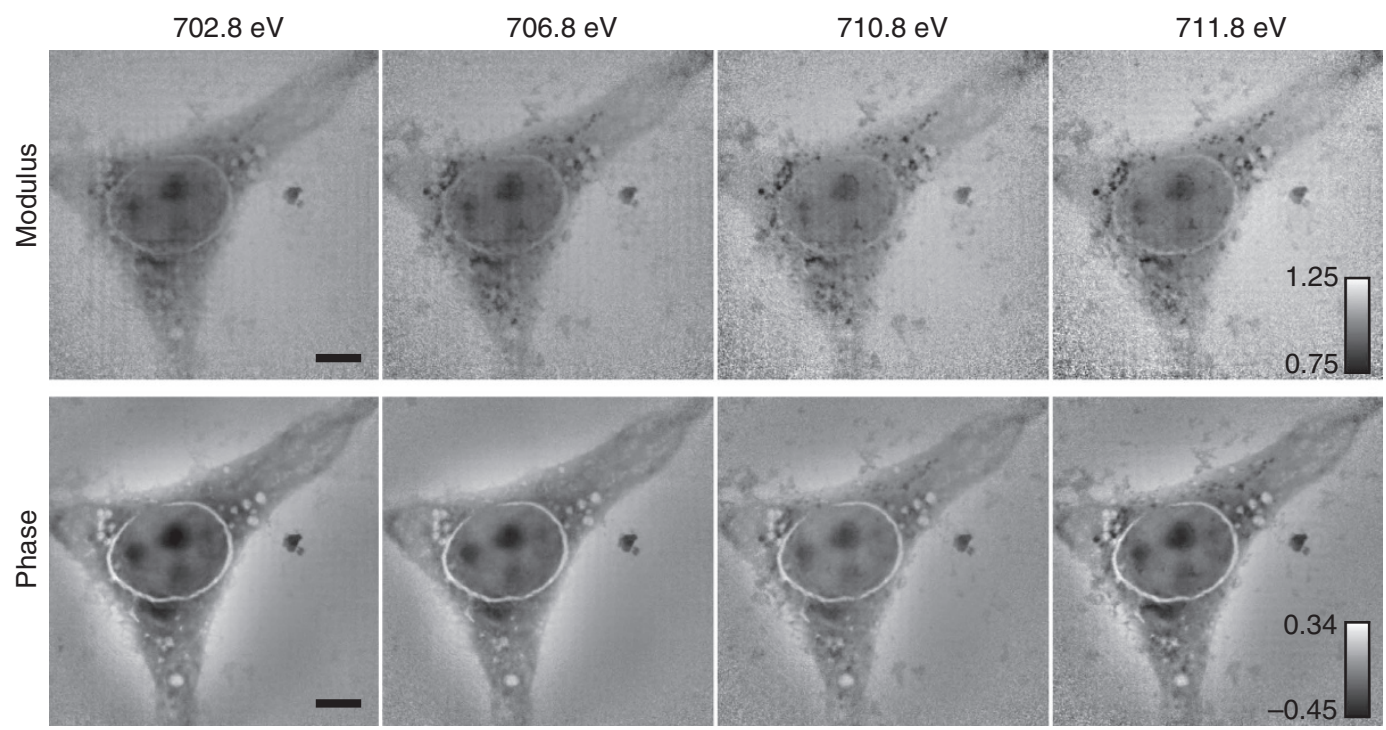

Figure 5 | Reconstructed modulus and phase images of a Balb/3T3 mouse fibroblast. The data were collected at X-ray beam energies across the iron $\mathrm{L}$ edge, showing the variation in contrast of the $\mathrm{CoFe}_{2} \mathrm{O}_{4}$ nanoparticles as a function of energy. Scale bar, $5 \mu \mathrm{m}$.

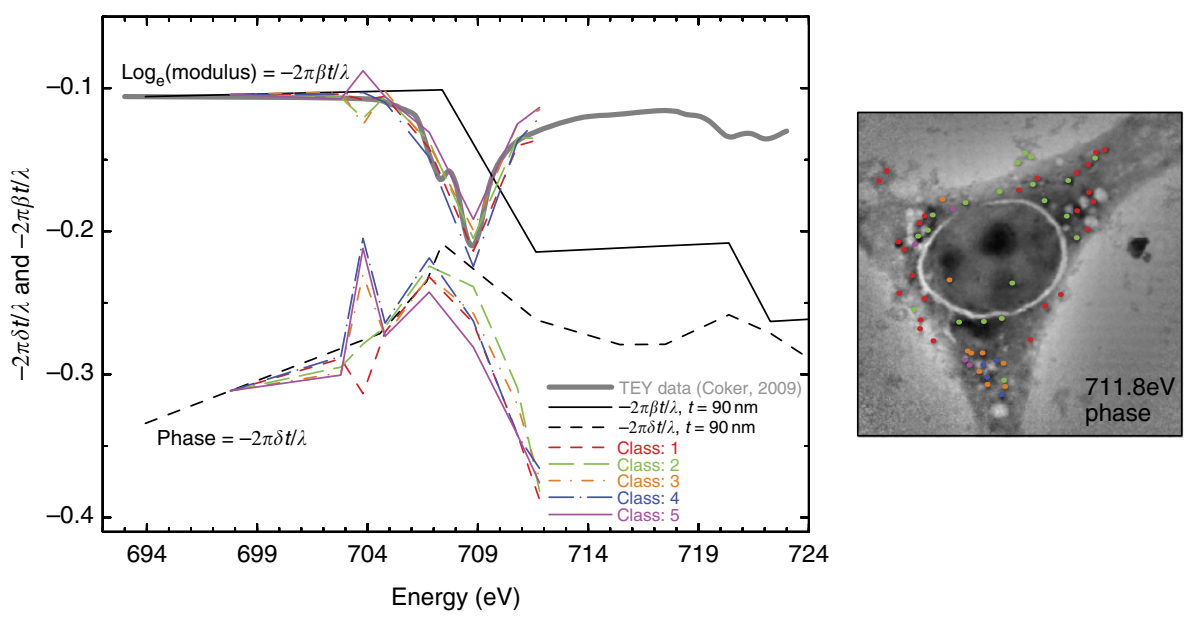

Figure 6 | Plot of the $\log _{e}$ (modulus) and phase image signals measured from the $\mathrm{CoFe}_{\mathbf{2}} \mathbf{O}_{\mathbf{4}}$ nanoparticles. The locations of the nanoparticle clusters are highlighted by coloured dots in the inset image (phase image at $711.8 \mathrm{eV}$ ). The curves show that five different classes of behaviour as a function of $\mathrm{X}$-ray energy were identified. For comparison, a scaled plot of the TEY signal from similar particles ${ }^{25}$ is overlaid on the $\log _{\mathrm{e}}$ (modulus) plots, along with curves calculated for a $90-\mathrm{nm}$ thick film of $\mathrm{CoFe}_{2} \mathrm{O}_{4}$ derived from the semi-empirical tabulation by Chantler ${ }^{22,23}$. 
optics. Our method is equally applicable at hard X-ray energies, and indeed the diffusing structure is easier to realize at these shorter wavelengths. We have also shown that the added complexity of the probe does not adversely affect the robustness and reliability of the reconstruction algorithm and that quantitative reconstructions of weakly scattering samples are possible using this experimental geometry. One difficulty we have encountered is that of suitably localizing the structured probe at the sample plane. The combination of a diffusing structure with a focusing optic could alleviate this issue.

Two previous studies have combined ptychography with beamenergy changes to realize chemically sensitive imaging. Beckers et al. ${ }^{10}$ used the absorption and phase changes at five energies across the oxygen $\mathrm{K}$ edge to distinguish between $2-\mu \mathrm{m}$ diameter spheres of polymethyl methacrylate and silicon dioxide. Takahashi et al. ${ }^{26}$ also made measurements of the phase signal at two energies below the Au M edge (around $11.9 \mathrm{keV}$ ) to study the distribution of $\mathrm{Au}$ in $\mathrm{Au} / \mathrm{Ag}$ nanoparticles. In our case, ptychographic data were collected at eight energies between 697.8 and $711.8 \mathrm{eV}$, and we have been able to show that both the modulus and the phase signals are sensitive to the chemical environment of the $\mathrm{Fe}$ atoms present in the $\mathrm{CoFe}_{2} \mathrm{O}_{4}$ nanoparticles. The general shape of the measured $\log _{e}$ (modulus) data matches well the TEY data taken from similar nanoparticles, while it is clear that the phase variations across the edge can give stronger and clearer features than the modulus data provided. It is also reassuring to note that the phase variation across the edge broadly follows the tabulated data from Chantler ${ }^{22,23}$, with the notable exception that the tabulated data do not show the very prominent pre-edge peak or dip that is measured experimentally around $704 \mathrm{eV}$.

From the shapes of the $\log _{e}$ (modulus) and phase variations across the edge, even on the relatively sparse set of data presented here, it is possible to see that the iron exists in a number of different chemical states. The X-ray magnetic circular dichroism measurements by Coker et al. ${ }^{25}$ suggested that at least three forms of iron were present: octahedrally coordinated $\mathrm{Fe}^{2+}$ and $\mathrm{Fe}^{3+}$ and tetrahedrally coordinated $\mathrm{Fe}^{3+}$. Calculations of the $2 p$ absorption spectra for iron with these symmetries indicate that the observed spectra will depend on $2 p-3 d$ and $3 d-3 d$ Coulomb and exchange interactions, $2 p$ and $3 d$ spin-orbit interactions and the crystal field acting on the $3 d$ states $^{27}$. The sparse nature of the spectral data in Fig. 6 does not allow a more detailed analysis of the components present in the different RoI classes. However, the distribution of the coloured dots in the inset image does show that particles in RoI class 2 lie predominantly in the perinuclear region, whereas those in class 1 lie further from the nucleus. The other three classes occur much less frequently, and almost all of them are found close together on the arm of the fibroblast in the lower part of the image field. It is not possible to determine from these limited data whether the changes in the chemical environment of the iron result directly from biodegradation within the cellular environment.

The ptychographic approach to imaging offers some important advantages for high-resolution X-ray spectromicroscopy. It provides modulus and phase reconstructions with a spatial resolution which is comparable to that achieved when the TwinMic microscope is operated as a conventional STXM, while the use of a relatively large probe on the sample allows a usefully large area to be covered in a raster of modest size, with no refocusing required when the incident X-ray energy was changed. At present, a rate-limiting step for our measurements is the time needed to readout the CCD (charge-coupled device) detector, but faster CCD systems are now available and it is reasonable to assume that much faster scans will be possible in future with an optimized system. We have also shown that near-edge measurements of the phase signal provide new information that complements and supplements that available from near-edge X-ray absorption fine structure (NEXAFS) measurements. NEXAFS imaging in the X-ray microscope has become a vital tool for the study and chemical characterization of nanoscale materials ${ }^{28,29}$, but hitherto the use of near-edge phase information has mostly relied on resonant soft X-ray reflectivity measurements ${ }^{30}$. Another promising approach involves the combination of multiple-wavelength anomalous diffraction with soft X-ray holography ${ }^{31}$, but this method requires a priori knowledge of the optical constants as a function of wavelength to provide high-resolution reconstructions that are sensitive to the elemental, chemical and magnetic state at the nanoscale. At present, there is a dearth of detailed experimental and theoretical data on the fine structure of phase variations near to absorption edges, and we believe that the ability of X-ray ptychography to provide direct, quantitative phase information in perfect registration with conventional NEXAFS data will open up a rich new branch of X-ray spectromicroscopy.

\section{Methods}

Experimental method. The soft X-ray diffuser consisted of a pseudo-random array of $\sim 40 \mathrm{~nm}$ diameter holes in a $140-\mathrm{nm}$ thick tungsten film in the beam path. The holes covered about a quarter of a $95 \times 95 \mu \mathrm{m}^{2}$ field in the tungsten film, with hole positions determined by the Mersenne Twister algorithm ${ }^{32}$ to avoid systematic correlations. Random hole patterns were supplied to our specification by Zoneplates Ltd, London, UK. The holes in the film acted as secondary sources with randomized phases, giving a diffuse, highly speckled beam downstream of the hole array. A $5-\mu \mathrm{m}$ pinhole placed in this beam produced a localized probe in the sample plane. The sample was scanned in small rasters transverse to the beam direction, with step sizes $\sim 1 \mu \mathrm{m}$, allowing a series of diffraction patterns to be recorded from overlapping areas using the X-ray sensitive CCD normally used for imaging in the TXM mode. The TXM camera was capable of outputting 16-bit images each of $1340 \times 1300$ pixels on a $20-\mu \mathrm{m}$ pitch. It was operated in accumulation mode, capturing 8 frames, each of $0.5 \mathrm{~s}$ dwell time, from each position in the raster. Memory limitations required that the recorded data be binned by a factor of 4 , and the resulting $335 \times 325$ pixel CCD frames were then cropped to $256 \times 256$ pixels. After each raster scan, a darkfield measurement was taken with the same camera settings and the beamline shutter closed, and this was subtracted from each diffraction pattern in the data set. No further efforts to mitigate noise in the data were undertaken.

Reconstruction procedure. Images were reconstructed using the ePIE method described by Maiden and Rodenburg ${ }^{3}$. The signal within a central disc of diameter 10 pixels was distorted by saturation in the binned diffraction patterns, but by allowing the signal to 'float' in this region (so that the diffraction constraint was not applied within this area during the course of the reconstruction), the ePIE method successfully reintroduced a smooth central peak into the diffraction data ${ }^{33}$.

It is better to use an irregular grid of illumination positions for ptychography to avoid periodic, ill-constrained features in the reconstruction. However, the STXM stage-control software was designed to perform only regular raster scans, so small random fluctuations in the specimen positions (within \pm 0.5 pixels) were introduced and varied during the reconstruction, thus breaking the regularity of the actual positions at the expense of a small loss in the attainable resolution. Averaging over the final 25 ePIE iterations further reduced noise in the reconstructed image. Because each ePIE iteration involves updating the current specimen estimate once for each of the $N$ recorded diffraction patterns, this averaging was taken over the final $25 \mathrm{~N}$ specimen estimates. The phase reconstructions are consistent in quality across the absorption edge and show very little noise at any energy. Although the modulus images are somewhat noisier, and they degrade slightly at energies below the absorption edge, the ePIE does not diverge.

Image analysis. Reconstructions at eight different energies were all re-sampled to the same pixel size, and the phase reconstructions packed into a three-dimensional stack that was aligned using the Multistackreg plug-in for ImageJ ${ }^{34,35}$. The same transformations were then applied to a similar stack of $\log _{e}$ (modulus) images to produce two mutually aligned sets of data.

The ptychographic image data were reconstructed with pixel sizes $\sim 80 \mathrm{~nm}$, which is almost two orders of magnitude smaller than the size of the illumination spot on the sample plane. As the features in the fibroblast cells are not of welldefined sizes and shapes, it is hard to get a precise determination of the spatial resolution in the reconstructions, but an estimate can be made by considering the signal profile across the boundary of the cell nucleus that appears as a bright ring in 
the phase reconstructions. The Straighten plug-in for Image ${ }^{34}$ was used to select arcs about 40 pixels wide that straddled part of the nuclear perimeter, the arcs were straightened using the method described in Kocsis et al. ${ }^{36}$ and then line profiles perpendicular to the arc were used to estimate the sharpness of the edge. The separation of $10-90 \%$ contours suggested that a spatial resolution $\sim 250 \mathrm{~nm}$ was achieved in the reconstructions, but in practice this should be considered an upperlimit (worst-case) estimate, because the cell boundary may not be sharply defined, and the process of arc selection, interpolation and alignment may well result in some broadening of the edge.

\section{References}

1. Guizar-Sicairos, M. \& Fienup, J. R. Phase retrieval with transverse translation diversity: a nonlinear optimization approach. Opt. Express 16, 7264-7278 (2008).

2. Thibault, P. et al. High-resolution scanning X-ray diffraction microscopy. Science 321, 379-382 (2008).

3. Maiden, A. M. \& Rodenburg, J. M. An improved ptychographical phase retrieval algorithm for diffractive imaging. Ultramicroscopy 109, 1256-1262 (2009).

4. Guizar-Sicairos, M. \& Fienup, J. R. Measurement of coherent X-ray focused beams by phase retrieval with transverse translation diversity. Opt. Express 17, 2670-2685 (2009).

5. Kewish, C. M. et al. Ptychographic characterization of the wavefield in the focus of reflective hard X-ray optics. Ultramicroscopy 110, 325-329 (2010).

6. Schropp, A. et al. Hard X-ray nanobeam characterization by coherent diffraction microscopy. Appl. Phys. Lett. 96, 091102 (2010).

7. Schropp, A. et al. Non-destructive and quantitative imaging of a nanostructured microchip by ptychographic hard X-ray scanning microscopy. J. Microsc. 241, 9-12 (2011).

8. Giewekemeyer, K. et al. Quantitative biological imaging by ptychographic X-ray diffraction microscopy. Proc. Natl Acad. Sci. USA 107, 529-534 (2010).

9. Giewekemeyer, K. et al. Ptychographic coherent X-ray diffractive imaging in the water window. Opt. Express 19, 1037-1050 (2011).

10. Beckers, M. et al. Chemical contrast in soft X-ray ptychography. Phys. Rev. Lett. 107, 208101 (2011).

11. Tripathi, A. et al. Dichroic coherent diffractive imaging. Proc. Natl Acad. Sci. USA 108, 13393-13398 (2011).

12. Dierolf, M. et al. Ptychographic X-ray computed tomography at the nanoscale. Nature 467, 436-439 (2010).

13. Guizar-Sicairos, M. et al. Phase tomography from X-ray coherent diffractive imaging projections. Opt. Express 19, 21345-21357 (2011).

14. Diaz, A. et al. Quantitative X-ray phase nanotomography. Phys. Rev. B 85, 020104 (2012)

15. Maiden, A. M., Rodenburg, J. M. \& Humphry, M. J. Optical ptychography: a practical implementation with useful resolution. Opt. Lett. 35, 2585-2587 (2010).

16. Guizar-Sicairos, M. et al. Role of the illumination spatial-frequency spectrum for ptychography. Phys. Rev. B 86, 100103 (2012).

17. Fenimore, E. E. \& Cannon, T. M. Coded aperture imaging with uniformly redundant arrays. Appl. Opt. 17, 337-347 (1978).

18. Marchesini, S. et al. Massively parallel X-ray holography. Nat. Photon. 2, 560-563 (2008).

19. Fannjiang, A. \& Liao, W. Phase retrieval with random phase illumination. J. Opt. Soc. Am. A 29, 1847-1859 (2012).

20. Kaulich, B. et al. TwinMic-a European twin X-ray microscopy station commissioned at ELETTRA. In Proc. 8th Int. Conf. X-ray Microscopy, IPAP Conf. Series Vol 7 (eds Aoki, S., Kagoshima, Y. \& Suzuki, Y.) 22-25 (Institute of Pure and Applied Physics, Tokyo, 2006).

21. Marmorato, P. et al. Cellular distribution and degradation of cobalt ferrite nanoparticles in Balb/3T3 mouse fibroblasts. Toxicol. Lett. 207, 128-136 (2011).

22. Chantler, C. T. Theoretical form factor, attenuation and scattering tabulation for $Z=1-92$ from $E=1-10 \mathrm{eV}$ to $E=0.4-1.0 \mathrm{MeV}$. J. Phys. Chem. Ref. Data 24, 71-643 (1995).

23. Chantler, C. T. Detailed tabulation of atomic form factors, photoelectric absorption and scattering cross section, and mass attenuation coefficients in the vicinity of absorption edges in the soft X-ray $(Z=30-36, Z=60-89$, $E=0.1-10 \mathrm{keV}$ ), addressing convergence issues of earlier work. J. Phys. Chem. Ref. Data 29, 597-1048 (2000).
24. Pattrick, R. et al. Cation site occupancy in spinel ferrites studied by X-ray magnetic circular dichroism: developing a method for mineralogists. Eur. J. Mineral 14, 1095-1102 (2002).

25. Coker, V. S. et al. Harnessing the extracellular bacterial production of nanoscale cobalt ferrite with exploitable magnetic properties. ACS Nano 3, 1922-1928 (2009).

26. Takahashi, Y. et al. Multiscale element mapping of buried structures by ptychographic X-ray diffraction microscopy using anomalous scattering. Appl. Phys. Lett. 99, 131905 (2011).

27. van der Laan, G. \& Kirkman, I. W. The $2 \mathrm{p}$ absorption spectra of $3 \mathrm{~d}$ transition metal compounds in tetrahedral and octahedral symmetry. J. Phys.: Condens. Matter 4, 4189-4204 (1992).

28. Urquhart, S. G. et al. NEXAFS spectromicroscopy of polymers: overview and quantitative analysis of polyurethane polymers. J. Electron Spectrosc. Relat. Phenom. 100, 119-135 (1999).

29. Ade, H. \& Stoll, H. Near-edge X-ray absorption fine-structure microscopy of organic and magnetic materials. Nat. Mater. 8, 281-290 (2009).

30. Wang, C. et al. Resonant soft X-ray reflectivity of organic thin films. J. Vac. Sci. Technol. A 25, 575-586 (2007).

31. Scherz, A. et al. Nanoscale imaging with resonant coherent X Rays: extension of multiple-wavelength anomalous diffraction to nonperiodic structures. Phys. Rev. Lett. 101, 076101 (2008).

32. Matsumoto, M. \& Nishimura, T. Mersenne twister: a 623-dimensionally equidistributed uniform pseudo-random number generator. ACM Trans. Model. Comput. Simul. 8, 3-30 (1998).

33. Maiden, A. M., Humphry, M. J., Zhang, F. \& Rodenburg, J. M. Superresolution imaging via ptychography. J. Opt. Soc. Am. A 28, 604-612 (2011).

34. Rasband, W. ImageJ. http://imagej.nih.gov/ij. National Institutes of Health, USA.

35. Thévenaz, P., Ruttimann, U. E. \& Unser, M. A pyramid approach to subpixel registration based on intensity. IEEE Trans. Image Processing 7, 27-41 (1998).

36. Kocsis, E., Trus, B. L., Steer, C. J., Bisher, M. E. \& Steven, A. C. Image averaging of flexible fibrous macromolecules: the clathrin triskelion has an elastic proximal segment. J. Struct. Biol. 107, 6-14 (1991).

\section{Acknowledgements}

This work was funded in part by the EPSRC Basic Technology Grant No. EP/E034055/1; 'ULTIMATE MICROSCOPY: Wavelength-Limited Resolution Without High Quality Lenses'. We thank G. Ceccone and J. Ponti (Joint Research Centre, Institute for Health and Consumer Protection, Ispra, Italy) for supplying the fibroblast cells, P. S. Charalambous of Zoneplates Ltd for manufacturing the random hole array and V. S. Coker of Manchester University for provding us with TEY data for the X-ray absorption of cobalt ferrite across the Fe L edge.

\section{Author contributions}

A.M.M. and G.R.M. devised the experiment and wrote the final version of the manuscript. A.M.M. and J.M.R. developed the idea of using a random diffuser to increase the phase diversity of the illumination, and G.R.M. designed the random hole diffuser suitable for use with soft X-rays. A.G. and B.K. reconfigured the Twinmic instrument and its control software to make the experiment possible, while A.G., A.M.M., B.K. and G.R.M. carried out the experiment. A.M.M. and J.M.R. developed the ePIE algorithm, and A.M.M. carried out the ptychographic image reconstructions. G.R.M. carried out the spectromicroscopy analysis of the image data. All authors contributed to the manuscript

\section{Additional information}

Competing financial interests: J.M.R. is a Director of and Shareholder in Phase Focus Ltd, a spin-out of the University of Sheffield intending to commercialize some aspects of the methods described in this paper. A.M.M. worked for the University of Sheffield when the work presented here was carried out, but is now employed by Phase Focus Ltd. The remaining authors declare no competing financial interests.

Reprints and permission information is available online at http://npg.nature.com/ reprintsandpermissions/

How to cite this article: Maiden, A.M. et al. Soft X-ray spectromicroscopy using ptychography with randomly phased illumination. Nat. Commun. 4:1669 doi: 10.1038/ ncomms2640 (2013). 